\title{
The Diagnostic, Prognostic, and Therapeutic Utility of Molecular Testing in a Patient with Waldenstrom's Macroglobulinemia
}

\author{
Collin K. Chin ${ }^{1}$ (D), Connull Leslie ${ }^{2}$, Carolyn S. Grove ${ }^{1,3}$, Chris Van Vliet ${ }^{2}$ and \\ Chan Yoon Cheah 1,3,* \\ 1 Department of Haematology, Sir Charles Gairdner Hospital and Pathwest Laboratory Medicine WA, \\ Nedlands 6009, Australia; Collin.Chin@health.wa.gov.au (C.K.C.); Carolyn.Grove@health.wa.gov.au (C.S.G.) \\ 2 Department of Anatomical Pathology, Pathwest Laboratory Medicine WA, Nedlands 6009, Australia; \\ Connull.Leslie@health.wa.gov.au (C.L.); Benjamin.VanVliet@health.wa.gov.au (C.V.V.) \\ 3 Medical School, University of Western Australia, Crawley 6009, Australia \\ * Correspondence: Chan.Cheah@health.wa.gov.au; Tel.: +61-8-6457-7600
}

Received: 30 August 2017; Accepted: 20 September 2017; Published: 22 September 2017

\begin{abstract}
The application of molecular genomics and our understanding of its clinical implications in the diagnosis, prognostication and treatment of lymphoproliferative disorders has rapidly evolved over the past few years. Of particular importance are indolent B-cell malignancies where tumour cell survival and proliferation are commonly driven by mutations involving the B-cell receptor and downstream signalling pathways. In addition, the increasing number of novel therapies and targeted agents have provided clinicians with new therapeutic options with the aim of exploiting such mutations. In this case report, we highlight one such success story involving the diagnostic impact of the $M Y D 88^{\mathrm{L} 265 \mathrm{P}}$ mutation in Waldenstrom's macroglobulinemia (WM), its prognostic implications and effect on choice of therapy in the era of novel therapies.
\end{abstract}

Keywords: Waldenstrom's macroglobulinemia; MYD88; Bruton's tyrosine kinase (BTK) inhibitors; molecular diagnostics

\section{Case Presentation}

An 89-year-old retired office worker with comorbidities including congestive cardiac failure and atrial fibrillation (on rivaroxaban) presented with a suprapubic mass and widespread lymphadenopathy. Further history revealed unintentional weight loss of $10 \mathrm{~kg}$ over six months and lethargy associated with a normocytic anaemia of $92 \mathrm{~g} / \mathrm{L}$ and IgM kappa paraproteinemia of $31 \mathrm{~g} / \mathrm{L}$. Positron-emission tomography computerized tomography (PET-CT) revealed extensive fluorodeoxyglucose (FDG) avid lymphadenopathy, bilateral pleural effusions, and diffuse activity in the bone marrow without evidence of high grade disease (maximum standardized uptake value $\left.\left[\mathrm{SUV}_{\max }\right] 2.4\right)$. Diagnostic thoracocentesis was negative for malignancy.

Core biopsy of an inguinal lymph node showed diffuse replacement of normal nodal architecture by small lymphocytes with interspersed plasmacytoid cells and mature plasma cells without follicular architecture or sheet-like large cell component. By immunohistochemistry, the lymphocytes were positive for cluster of differentiation 20 (CD20), B-cell lymphoma 2 (BCL2), CD10, CD23 (minor subset), Immunoglobulin D (IgD), Immunoglobulin M (IgM), kappa light chains and negative for CD3, CD5, B-cell lymphoma 6 (BCL6), Lim domain only 2 (LMO2), cyclin D1, SRY-Box 11 (SOX11), Epstein-Barr encoding region (EBER), and lambda light chains (Figure 1A,B). CD138 and Multiple Myeloma Oncogene 1 (MUM1) highlight a subpopulation of mature plasma cells (Figure 1C). The Ki-67 proliferation index was $<10 \%$. A bone marrow biopsy was hypercellular with heavy diffuse and 
interstitial infiltrate of small lymphocytes with similar morphology and immunophenotype, with some preservation of background trilineage haematopoiesis (Figure 1D). Both the lymphocytes and plasma cells were CD79a positive and occupied about 30\% of the marrow space. Given the lack of follicularity or large cell component, the differential diagnoses favoured nodal marginal zone lymphoma or lymphoplasmacytic lymphoma although positivity for CD10 raised the possibility of diffuse patterned follicular lymphoma.
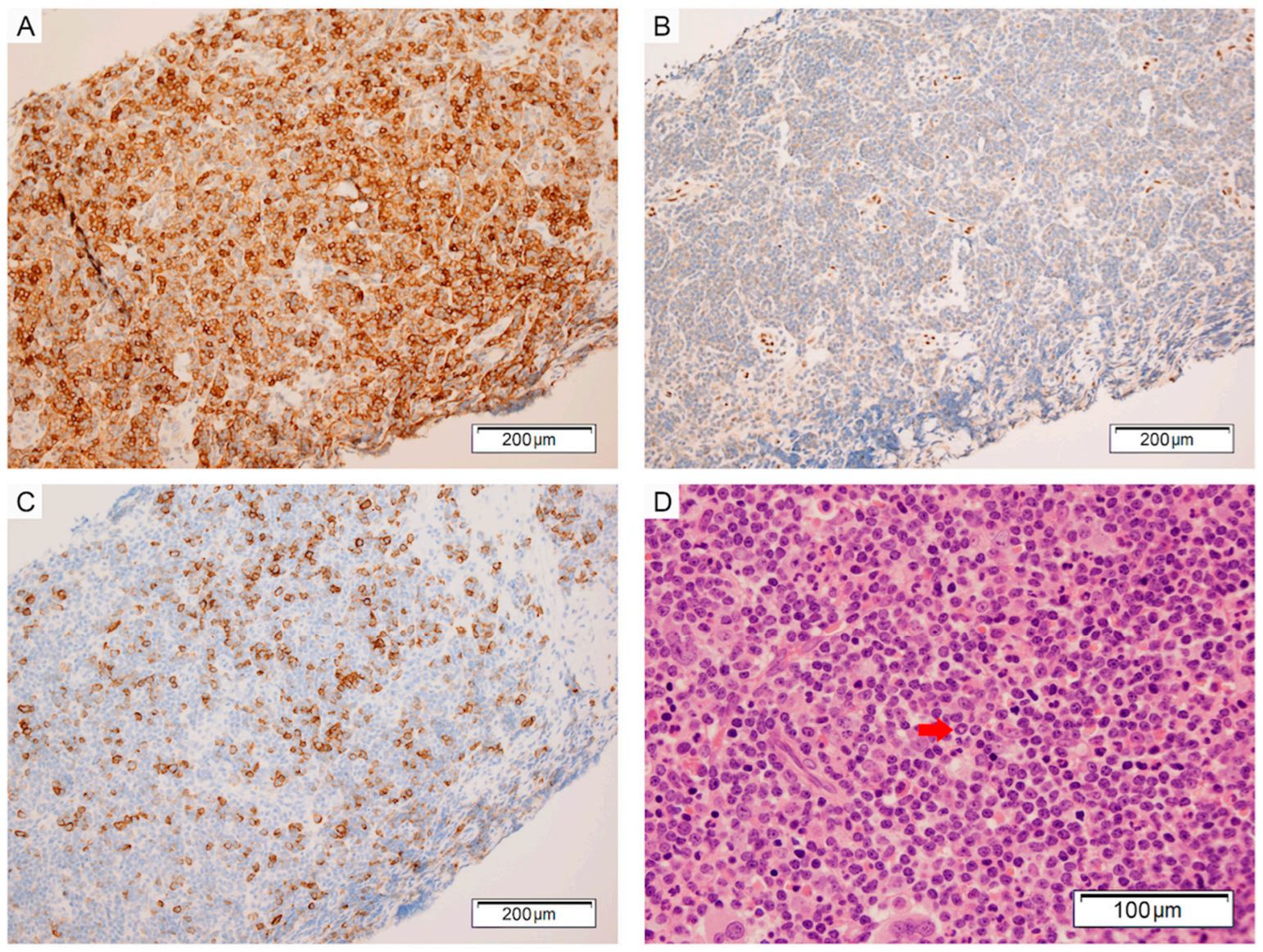

Figure 1. (A-C) Inguinal lymph node core biopsy; (A) CD10 is positive in the lymphoma cells; (B) LMO2 is negative in the lymphoma cells; (C) CD138 is positive in mature plasma cells; (D) Bone marrow trephine containing a diffuse infiltrate of small mature lymphocytes with interspersed plasma cells. Note the Dutcher body (arrow).

Testing for MYD88 ${ }^{\mathrm{L} 265 \mathrm{P}}$ mutation was performed on formalin fixed paraffin embedded tissue by digital polymerase chain reaction (dPCR) from the lymph node biopsy and bone marrow trephine, which demonstrated the MYD88 ${ }^{\mathrm{L} 265 \mathrm{P}}$ mutation present at an allele frequency of $46 \%$ and $0.69 \%$, respectively. Confirmatory testing for MYD88 and CXCR4 mutation was performed on bone marrow aspirate using bi-directional Sanger sequencing on exon 5 of MYD88 and C1013G/CXCR4 mutation on chromosome 2q21, respectively. MYD8 $8^{\mathrm{L} 265 \mathrm{P}}$ and CXCR4 $4^{\mathrm{WT}}$ (WT = wild type) were subsequently confirmed. The final diagnosis was lymphoplasmacytic lymphoma (LPL) with aberrant CD10 expression, $M Y D 88^{\mathrm{L} 265 \mathrm{P}}$ mutation and $C X C R 4^{\mathrm{WT}}$. Taken in conjunction with the IgM kappa serum paraprotein, the findings were consistent with Waldenstrom's macroglobulinemia (WM).

In this case detection of the MYD88 ${ }^{\mathrm{L} 265 \mathrm{P}}$ mutation by digital PCR in conjunction with clinical and morphologic features confirmed the diagnosis of WM despite aberrant CD10+ expression on immunohistochemistry. Although lacking symptoms of hyperviscosity, peripheral neuropathy, or other paraneoplastic sequelae, the patient's symptomatic anaemia provided reason to commence therapy. 
Given the patient's age, comorbidities, and genomic profile, he was offered enrolment in a clinical trial with a second generation BTK inhibitor with careful clinical monitoring due to his atrial fibrillation and rivaroxaban. Treatment has been well tolerated and initial response assessment is ongoing.

\section{Discussion}

\subsection{Diagnostic Relevance of MYD88 L265P}

MYD88 is an adaptor protein that mediates toll and interleukin (IL)-1 receptor signaling. $M Y D 88^{\mathrm{L} 265 \mathrm{P}}$ is a gain of function mutation that drives lymphomagenesis by promoting cell survival through nuclear factor- $\mathrm{kB}$ (NF- $\mathrm{kB}$ ). The mutation allows spontaneous assembly of a protein complex containing interleukin-1 receptor-associated kinases (IRAK1 and IRAK4), leading to increased IRAK kinase activity and downstream NF- $\kappa B$ activation [1]. Additionally, MYD88 ${ }^{\mathrm{L} 265 \mathrm{P}}$ enhances Bruton's tyrosine kinase (BTK) phosphorylation resulting in the downstream activation of NF- $\mathrm{kB}$ independent of IRAK/IRAK4 signaling [2]. MYD88 ${ }^{\mathrm{L} 265 \mathrm{P}}$ is a recurring somatic mutation in WM and LPL and occurs in approximately $90 \%$ of cases [3]. MYD $88^{\mathrm{L} 265 \mathrm{P}}$ can also be detected by highly sensitive assays in IgM MGUS (monoclonal gammopathy of undetermined significance) and WM patients undergoing treatment [4]. PCR is the preferred method for detecting the $M Y D 88^{\mathrm{L} 265 \mathrm{P}}$ mutation due to its high sensitivity, rapidity, low cost, and ability to detect point mutations as compared to fluorescence in situ hybridization (FISH) studies which have a limit of resolution of 50 kilobases. Sanger sequencing for non-L265P MYD88 mutations has been used for patients who are negative for MYD88 ${ }^{\mathrm{L} 265 \mathrm{P}}$ by allele-specific polymerase chain reaction (AS-PCR) as other types of MYD88 mutations have also been demonstrated in LPL, with a recent prospective study identifying 2 out of 57 patients with MYD88 mutations harbouring a non-L265P mutation [5]. Single nucleotide polymorphism-based array (SNPa) may also be used to detect non-L265P mutations. A French study involving 31 patients with WM identified copy number alterations or loss of heterozygosity in $64 \%$, although the role of these genetic aberrations in the diagnosis and treatment of WM still remains unclear [6]. Sanger sequencing remains the gold standard and is more accessible, more affordable, and quicker to perform.

MYD88 mutation testing is of most value in diagnosing small B cell lymphomas with plasmacytic differentiation in which LPL is a strong possibility, as a positive result is highly suggestive of LPL [7]. It is important to recognise that the MYD88 ${ }^{\mathrm{L} 265 \mathrm{P}}$ mutation is not specific as it is found in a small proportion of splenic marginal zone lymphomas $(\sim 15 \%)[8,9]$, and rarely in other small B cell lymphomas $[7,9,10]$. It is uncertain whether this represents secondary acquisition of the MYD88 mutation or if this is an unusual primary driving event in these tumours [7]. In addition, MYD88 $265 \mathrm{P}$ mutation is the most frequent genomic abnormality in diffuse large $B$ cell lymphoma (DLBCL) activated B-cell-like (ABC) subtype, as it is detected in $40 \%$ of cases [11].

\subsection{Prognostic Implications of MYD88 in WM}

In the era of novel agents, Treon et al. have reported three distinct genomic groups of WM differentiated by MYD 88 and CXCR4 mutation status. These groups showed significantly different clinical manifestations and overall survival (OS) [12]. MYD88 ${ }^{\mathrm{WT}}$ status was shown to have lower BM disease burden but significantly inferior overall survival ( 4.7 years vs. $>10$ years; $p=0.0018$ ). In contrast, MYD88 ${ }^{\mathrm{L} 265 \mathrm{P}}$ CXCR4 ${ }^{\mathrm{WHIM} / \mathrm{NS}}$ (WHIM = syndrome associated with warts, hypogammaglobulinaemia, infections, and myelokathexis; NS = nonsense mutation) was associated with significantly higher $\mathrm{BM}$ involvement, higher IgM paraproteinaemia and symptomatic disease, including hyperviscosity $(p=0.03) . M Y D^{\mathrm{L} 265 \mathrm{P}} C X C R 4^{\mathrm{WHIM} / \mathrm{FS}}$ (FS = frameshift mutation) and $M Y D^{\mathrm{L} 265 \mathrm{P}} C X C R 4^{\mathrm{WT}}$ were associated with intermediate disease burden. The dependence of WM tumour cells on activation of NF- $\mathrm{KB}$ by the BTK and IRAK pathways has been demonstrated by the significant activity seen in pre-clinical and clinical studies using IRAK1/4, BTK, and NF-kB inhibitors [13-18]. Although BTK inhibition has been found to be highly effective in the treatment of WM, the efficacy is influenced by MYD88 mutation status [5]. In patients with mutated MYD88 but CXCR4 ${ }^{\mathrm{WT}}$, the overall response 
rate (ORR) to the BTK inhibitor ibrutinib is $100 \%$ with a $91.7 \%$ major response rate. Patients with wild-type MYD88 have significantly inferior outcomes with an ORR of $60 \%(p=0.005)$ and no major responses $(p<0.001)$. In addition, patients with non-L265P MYD88 mutations (e.g., S243N, M232T) have been shown to respond favourably to ibrutinib compared to wild-type MYD88 [5]. MYD88 mutation status has not been shown to be associated with inferior outcomes when treated with chemoimmunotherapy [19].

\subsection{Current and Future Treatment Approaches for Patients with WM}

Outside the setting of a clinical trial, chemoimmunotherapy remains the treatment of choice in the treatment-naïve patients with LPL. No standard therapy exists. However, rituximab, cyclophosphamide, and dexamethasone (RCD) is highly effective with a $96 \%$ ORR and $87 \%$ major response rate in treatment naïve $\mathrm{WM}$, and $87 \%$ ORR and $68 \%$ major response in the relapsed setting. These responses are relatively durable with a 2-year PFS of $67 \%$ and median PFS of 34 months in treatment naive patients [19]. Rummel et al. randomized 41 patients with WM to rituximab in combination with bendamustine (BR) or cyclophosphamide, doxorubicin, vincristine and prednisolone (R-CHOP) and found a significant difference in median progression free survival (PFS; 69.5 versus 28.1 months, $p=0.0033$ ) [20]. However, in patients considered unsuitable for these approaches, other treatments are needed.

Ibrutinib has been shown to be highly active in the relapsed setting (2-year PFS 69\%, median PFS not reached) [5]. Furthermore, ibrutinib has been shown to be well tolerated with less frequent grade $\geq 3$ neutropenia ( $14 \%$ vs. $20 \%$ ) and infection (none directly related to ibrutinib vs. $3 \%$ ) but with a higher risk of grade $\geq 3$ thrombocytopenia ( $13 \%$ vs. $7 \%$ ), grade $\geq 2$ bleeding $(6 \%)$ and atrial fibrillation $(5 \%)$ when compared to chemoimmunotherapy $[19,20]$. Notably, ibrutinib has not been shown to induce a paraprotein 'flare' as is associated with rituximab but does induce a peripheral blood lymphocytosis [18]. A phase 1 study of BGB-3111, a highly-specific irreversible second generation BTK inhibitor with greater selectivity than ibrutinib for BTK has shown significant anti-tumor activity in WM. Tam et al. treated 31 patients with relapsed/refractory and previously untreated WM with BGB-3111 and reported a $92 \%$ ORR and $83 \%$ major response rate after a median follow-up of 7.6 months (2-21 months) [21]. BGB-3111 was well tolerated with $71 \%$ of patients reporting no drug related AE (>grade 1) within the first 12 weeks of therapy and no cases of serious haemorrhage ( $\geq$ grade 3 or CNS haemorrhage of any grade). Analysis of response by genomic characteristics including MYD88 and CXCR4 mutational status are awaited. Patients with heavily pre-treated or chemorefractory disease should be offered therapy with BTK inhibitors when these agents are available.

Venetoclax, a highly selective BCL-2 inhibitor has also been shown to have significant activity in WM. A phase 1 study by Davids et al. [22] identified 106 patients with relapsed/refractory non-Hodgkin's lymphoma (NHL) treated with venetoclax monotherapy which included four patients with WM. All patients with WM responded (all PR, no CRs) with the duration of response ranging between 11 to 41 months [22]. Treatment was reasonably well tolerated. Grade $\geq 3$ events were uncommon with anaemia (15\%), neutropenia (11\%), thrombocytopenia (9\%) the most frequent and $3 \%$ of patients developing laboratory tumour lysis syndrome with no clinical sequelae. Clinical trials investigating the potential synergistic combinations of venetoclax and BTK inhibitors in other subtypes of indolent NHL are currently in progress (NCT02956382, NCT03112174).

\section{Conclusions}

MYD88 mutation status is useful in the diagnosis, prognostication and prediction of response to targeted therapy in LPL. Further studies of MYD88 and its downstream signalling pathways are required to better understand mechanisms of disease resistance to BTK inhibitors. Ongoing prospective studies will hopefully determine the role of these highly effective agents in patients with WM, including in previously untreated patients. 
Author Contributions: C.K.C. wrote the manuscript; C.L. and C.V.V. provided pathology reports, images \& co-wrote the section on diagnostic relevance; C.S.G. reviewed the manuscript; C.Y.C. designed the paper and reviewed the manuscript.

Conflicts of Interest: The authors declare no conflict of interest.

\section{References}

1. Ngo, V.N.; Young, R.M.; Schmitz, R.; Jhavar, S.; Xiao, W.; Lim, K.H.; Kohlhammer, H.; Xu, W.; Yang, Y.; Zhao, H.; et al. Oncogenically active MYD88 mutations in human lymphoma. Nature 2011, 470, 115-119. [CrossRef] [PubMed]

2. Yang, G.; Zhou, Y.; Liu, X.; Xu, L.; Cao, Y.; Manning, R.J.; Patterson, C.J.; Buhrlage, S.J.; Gray, N.; Tai, Y.T.; et al. A mutation in MYD88 (1265p) supports the survival of lymphoplasmacytic cells by activation of bruton tyrosine kinase in waldenstrom macroglobulinemia. Blood 2013, 122, 1222-1232. [CrossRef] [PubMed]

3. Treon, S.P.; Xu, L.; Yang, G.; Zhou, Y.; Liu, X.; Cao, Y.; Sheehy, P.; Manning, R.J.; Patterson, C.J.; Tripsas, C.; et al. MYD88 L265P somatic mutation in Waldenstrom's macroglobulinemia. N. Engl. J. Med. 2012, 367, 826-833. [CrossRef] [PubMed]

4. Xu, L.; Hunter, Z.R.; Yang, G.; Zhou, Y.; Cao, Y.; Liu, X.; Morra, E.; Trojani, A.; Greco, A.; Arcaini, L.; et al. MYD88 L265P in Waldenstrom macroglobulinemia, immunoglobulin M monoclonal gammopathy, and other B-cell lymphoproliferative disorders using conventional and quantitative allele-specific polymerase chain reaction. Blood 2013, 121, 2051-2058. [CrossRef] [PubMed]

5. Treon, S.P.; Xu, L.; Hunter, Z. MYD88 mutations and response to ibrutinib in Waldenstrom's macroglobulinemia. N. Engl. J. Med. 2015, 373, 584-586. [CrossRef] [PubMed]

6. Poulain, S.; Roumier, C.; Galiegue-Zouitina, S.; Daudignon, A.; Herbaux, C.; Aiijou, R.; Lainelle, A.; Broucqsault, N.; Bertrand, E.; Manier, S.; et al. Genome wide snp array identified multiple mechanisms of genetic changes in Waldenstrom macroglobulinemia. Am. J. Hematol. 2013, 88, 948-954. [CrossRef] [PubMed]

7. Swerdlow, S.H.; Kuzu, I.; Dogan, A.; Dirnhofer, S.; Chan, J.K.; Sander, B.; Ott, G.; Xerri, L.; Quintanilla-Martinez, L.; Campo, E. The many faces of small B cell lymphomas with plasmacytic differentiation and the contribution of MYD88 testing. Virchows Arch. 2016, 468, 259-275. [CrossRef] [PubMed]

8. Martinez-Lopez, A.; Curiel-Olmo, S.; Mollejo, M.; Cereceda, L.; Martinez, N.; Montes-Moreno, S.; Almaraz, C.; Revert, J.B.; Piris, M.A. MYD88 (L265P) somatic mutation in marginal zone B-cell lymphoma. Am. J. Surg. Pathol. 2015, 39, 644-651. [CrossRef] [PubMed]

9. Varettoni, M.; Arcaini, L.; Zibellini, S.; Boveri, E.; Rattotti, S.; Riboni, R.; Corso, A.; Orlandi, E.; Bonfichi, M.; Gotti, M.; et al. Prevalence and clinical significance of the MYD88 (L265P) somatic mutation in Waldenstrom's macroglobulinemia and related lymphoid neoplasms. Blood 2013, 121, 2522-2528. [CrossRef] [PubMed]

10. Martinez-Trillos, A.; Pinyol, M.; Navarro, A.; Aymerich, M.; Jares, P.; Juan, M.; Rozman, M.; Colomer, D.; Delgado, J.; Gine, E.; et al. Mutations in TLR/MYD88 pathway identify a subset of young chronic lymphocytic leukemia patients with favorable outcome. Blood 2014, 123, 3790-3796. [CrossRef] [PubMed]

11. Taniguchi, K.; Takata, K.; Chuang, S.S.; Miyata-Takata, T.; Sato, Y.; Satou, A.; Hashimoto, Y.; Tamura, M.; Nagakita, K.; Ohnishi, N.; et al. Frequent MYD88 L265P and CD79B mutations in primary breast diffuse large B-cell lymphoma. Am. J. Surg. Pathol. 2016, 40, 324-334. [CrossRef] [PubMed]

12. Treon, S.P.; Cao, Y.; Xu, L.; Yang, G.; Liu, X.; Hunter, Z.R. Somatic mutations in MYD88 and CXCR4 are determinants of clinical presentation and overall survival in Waldenstrom macroglobulinemia. Blood 2014, 123, 2791-2796. [CrossRef] [PubMed]

13. Lim, K.H.; Romero, D.L.; Chaudhary, D. IRAK4 kinase as a novel therapeutic agent in the ABC subtype of diffuse large B cell lymphoma. Blood 2012, 120, 62.

14. Treon, S.P.; Ioakimidis, L.; Soumerai, J.D.; Patterson, C.J.; Sheehy, P.; Nelson, M.; Willen, M.; Matous, J.; Mattern, J., 2nd; Diener, J.G.; et al. Primary therapy of Waldenstrom macroglobulinemia with bortezomib, dexamethasone, and rituximab: Wmctg clinical trial 05-180. J. Clin. Oncol. 2009, 27, 3830-3835. [CrossRef] [PubMed]

15. Treon, S.P.; Tripsas, C.K.; Meid, K.; Kanan, S.; Sheehy, P.; Chuma, S.; Xu, L.; Cao, Y.; Yang, G.; Liu, X.; et al. Carfilzomib, rituximab, and dexamethasone (CaRD) treatment offers a neuropathy-sparing approach for treating Waldenstrom's macroglobulinemia. Blood 2014, 124, 503-510. [CrossRef] [PubMed] 
16. Dimopoulos, M.A.; Garcia-Sanz, R.; Gavriatopoulou, M.; Morel, P.; Kyrtsonis, M.C.; Michalis, E.; Kartasis, Z.; Leleu, X.; Palladini, G.; Tedeschi, A.; et al. Primary therapy of Waldenstrom macroglobulinemia (WM) with weekly bortezomib, low-dose dexamethasone, and rituximab (BDR): Long-term results of a phase 2 study of the european myeloma network (EMN). Blood 2013, 122, 3276-3282. [CrossRef] [PubMed]

17. Burhlage, S. Kinome targets and inhibitors. In Proceedings of the 8th International Workshop on Waldenstrom's Macroglobulinaemia, London, UK, 13-17 August 2014.

18. Treon, S.P.; Tripsas, C.K.; Meid, K.; Warren, D.; Varma, G.; Green, R.; Argyropoulos, K.V.; Yang, G.; Cao, Y.; $\mathrm{Xu}$, L.; et al. Ibrutinib in previously treated Waldenstrom's macroglobulinemia. N. Engl. J. Med. 2015, 372, 1430-1440. [CrossRef] [PubMed]

19. Paludo, J.; Abeykoon, J.P.; Kumar, S.; Shreders, A.; Ailawadhi, S.; Gertz, M.A.; Kourelis, T.; King, R.L.; Reeder, C.B.; Leung, N.; et al. Dexamethasone, rituximab and cyclophosphamide for relapsed and/or refractory and treatment-naive patients with Waldenstrom macroglobulinemia. Br. J. Haematol. 2017. [CrossRef] [PubMed]

20. Rummel, M.J.; Niederle, N.; Maschmeyer, G.; Banat, G.A.; von Grunhagen, U.; Losem, C.; Kofahl-Krause, D.; Heil, G.; Welslau, M.; Balser, C.; et al. Bendamustine plus rituximab versus chop plus rituximab as first-line treatment for patients with indolent and mantle-cell lymphomas: An open-label, multicentre, randomised, phase 3 non-inferiority trial. Lancet 2013, 381, 1203-1210. [CrossRef]

21. Tam, C.S.; Trotman, J.; Opat, S.; Marlton, P.; Cull, G.; Simpson, D.; Ku, M.; Ritchie, D.; Verner, E.; Ratnasingam, S.; et al. High major response rate, including very good partial responses (VGPR), in patients (pts) with Waldenstrom macroglobulinemia (WM) treated with the highly specific BTK inhibitor bgb-3111: Expansion phase results from an ongoing phase I study. Blood 2016, 128, 1216.

22. Davids, M.S.; Roberts, A.W.; Seymour, J.F.; Pagel, J.M.; Kahl, B.S.; Wierda, W.G.; Puvvada, S.; Kipps, T.J.; Anderson, M.A.; Salem, A.H.; et al. Phase I first-in-human study of venetoclax in patients with relapsed or refractory non-hodgkin lymphoma. J. Clin. Oncol. 2017, 35, 826-833. [CrossRef] [PubMed]

(C) 2017 by the authors. Licensee MDPI, Basel, Switzerland. This article is an open access article distributed under the terms and conditions of the Creative Commons Attribution (CC BY) license (http:// creativecommons.org/licenses/by/4.0/). 